\title{
Nonadditive genetic structuring of morphometric variation in relation to a population bottleneck
}

\author{
EDWIN H. BRYANT* \& LISA M. MEFFERT \\ Department of Biology, University of Houston, Houston, TX 77204-5513, U.S.A.
}

\begin{abstract}
The genetic architectures of four morphometric traits in the housefly, Musca domestica L., were analysed using a paternal half-sib design on a large outbred base population and on two populations each established with two pairs of flies from the base population. By including parents with the offspring in the analysis, phenotypic variance was subdivided into components for additive, dominance, additive-by-additive epistasis and environmental variation. In relation to the nonbottlenecked base population, narrow sense hertitability was lower in the bottlenecked populations, largely because of an increase in the level of the environmental component of variance. Averaged over the four traits in the nonbottlenecked base population, nonadditive components contributed nearly 60 per cent of the phenotypic variance, with more than half of this being attributable to epistasis. Two of the four traits showed reduced additive genetic variance in the bottlenecked lines, whereas two of the traits showed increased additive genetic variance. A major difference in the genetic architecture between traits showing these two disparate bottleneck effects was the presence of epistasis in the nonbottlenecked base population for the traits which showed an increase in the additive component. Overall, there was a significant positive relationship between the initial percentage contribution of epistasis in the base population and the gain in the additive component in the bottleneck lines, as predicted by models incorporating additive-by-additive epistasis.
\end{abstract}

Keywords: bottlenecks, epistasis, quantitative genetics.

\section{Introduction}

The ways that biological traits are genetically structured can have profound effects on their evolutionary responses. Wright's shifting balance theory, for example (Wright, 1931, 1940), rests critically upon traits being structured by interactions among loci (i.e. by epistasis). The effects of population bottlenecks on quantitative traits influenced by nonadditive genetic processes may be quite complex, often leading to increases in the additive components of genetic variance, in direct contrast to the expected loss for traits with strictly additive gene action (Robertson, 1952; Cockerham \& Tachida, 1988; Goodnight, 1987, 1988, 1995; Tachida \& Cockerham, 1989a,b; Willis \& Orr, 1993).

Theoretically, many polygenic traits are expected to involve nonadditive components of variance as a consequence of either directional selection (A.

${ }^{*}$ Correspondence.
Robertson, 1955; F. W. Robertson, 1955) or stabilizing selection (Wright, 1935; Gimelfarb, 1989; Hastings, 1989). The prevalence of inbreeding depression for fitness-related traits attests to the widespread occurrence of dominance (e.g. Charlesworth \& Charlesworth, 1987). However, only a few cases of epistasis for quantitative traits have been clearly documented (e.g., see reviews by Kearsey \& Kojima, 1967; Hedrick et al., 1978; Barker, 1979), suggesting that epistasis is either rare or simply difficult to detect by most experimental procedures (e.g. Miller et al., 1963; Robertson, 1967). Our previous work has shown that additive genetic variance for morphometric traits in the housefly increased in response to population bottlenecks (Bryant et al., 1986; Bryant \& Meffert, 1993), thus indirectly implicating nonadditive components of variance in the ancestral population. However, we did not provide direct evidence that epistasis affected the traits, although the decline in trait means after the first bottleneck suggested that dominance may have been 
involved (Bryant et al., 1986; Willis \& Orr, 1993). If nonadditive components of genetic variances are responsible for previously observed increases in additive genetic variance after a bottleneck, as suggested by Bryant et al. (1986) and Bryant \& Meffert (1993), then the traits should exhibit substantial nonadditive components of genetic variation. This paper then provides estimates of genetic components in an outbred population of houseflies, and, by comparing these components with those in bottlenecked populations, focuses on the putative role that nonadditive components of genetic variance may play in the genetic responses of populations to a bottleneck.

The genetic basis of quantitative traits can be assayed in several ways: by substituting whole chromosomes (e.g. Robertson, 1954; Kearsey \& Kojima, 1967), analysing crosses and backcrosses between populations (e.g. Mather \& Jinks, 1982; Hard et al., 1992), including methods of QTL mapping (e.g. Lander \& Botstein, 1989), or analysing variance components in analysis of variance designs (e.g. Cockerham, 1956; Miller et al., 1963; Cowley et al., 1986; Becker, 1988; Cowley \& Atchley, 1988). In a prior study, we used crosses on lines that had undergone artificial selection for altered morphometric shape to show that the basis of the selectional response in shape involved dominance and epistasis (Bryant \& Meffert, 1995). The classical hierarchical half-sib design can estimate dominance but not epistasis (e.g. Riska et al., 1985; Becker, 1988; Cowley \& Atchley, 1988), but the inclusion of parents in the analysis allows for simultaneous estimation of some epistatic components as well (e.g. Becker, 1988). The current study, based on a half-sib design, extends our previous results on the genetic basis of selectional response to provide direct evidence that the morphometric traits under study have substantial nonadditive genetic structures that affects their genetic responses to a population bottleneck.

\section{Materials and methods}

\section{Data collection}

An outbred population of houseflies (Musca domestica L.) was established in the laboratory from a single collection of several hundred flies taken from a refuse site in Pasadena, TX. The initial sample of flies increased to a standard husbandry population size of at least 2000 within a few generations, after which the experimental design was initiated. From this nonbottlenecked (base) population (N), two bottlenecked lines $\left(B_{1}\right.$ and $\left.B_{2}\right)$ were each derived using offspring from two randomly sampled pairs of flies (creating a two-pair bottleneck) and allowed to flush to the standard population size before initiation of the genetic analyses. During the same period the base (nonbottlenecked) population was maintained at the standard husbandry size.

The paternal half-sib design consisted of mating each of a number of males to two virgin females and, from each of these females, three female offspring were sampled. The nonbottlenecked base population was assayed for a total of 431 sires $(862$ dams and 2586 offspring): for bottlenecked lines $B_{1}$ and $B_{2}$, a total of 162 sires (324 dams and 972 progeny) and 212 sires (424 dams and 1272 offspring) were assayed, respectively. All flies were reared under standard conditions of 80 eggs per $18 \mathrm{~g}$ larval medium, $27^{\circ} \mathrm{C}$, and a $12: 12$ light:dark cycle. Flies were pinned and measured with an ocular micrometer on a binocular microscope. Four traits (wing length, inner-eye separation, scutellum length and metatibia length; see Bryant, 1977, for detailed descriptions of these traits) were selected based on their separate associations with the first four principal axes of the additive genetic covariance matrix for the larger set of (log-transformed) morphometric traits of previous studies (Bryant \& Meffert, 1988, 1990). Consequently, the traits represent essentially four independent axes of genetic variation for morphology. All traits were transformed to natural logarithms before analysis to equilibrate variation caused by size differences among traits (Bryant, 1986) and for conformation to normality (using the Kolmogorov-Smirnoff test in SPSsx; Nie et al., 1975).

\section{Data analysis}

The design consisted of a hierarchical analysis of variance, whose mean squares (and variance components) thus constituted the observational components from which the causal (genetic) components were estimated. Following the recommendations of Lush (1940) and Falconer (1989, p. 172), covariances of offspring and their mothers were calculated within sires. Narrow sense heritabilities $\left(h^{2}\right)$ were computed from the variance component among sires (Falconer, 1989):

$h^{2}=4 \sigma_{\mathrm{S}}^{2} / \sigma_{\mathrm{T}}^{2}$,

where the total phenotypic variance $\left(\sigma_{\mathrm{T}}^{2}\right)$ is the sum of the variance components for sires $\left(\sigma_{S}^{2}\right)$, dams $\left(\sigma_{\mathrm{D}}^{2}\right)$ and within progenies $\left(\sigma_{\mathrm{W}}^{2}\right)$. Standard errors for herit- 
abilities were obtained using Dickerson's method outlined in Becker (1988).

Because of the balanced hierarchical design (equal number of progeny per female and equal number of females per male) maximum likelihood analyses of variance (Shaw, 1987) were unnecessary, and a weighted least squares approach was used with the mean squares from analysis of variance as dependent variables and the causal (genetic) components as independent variables (e.g. Draper \& Smith, 1981; Cockerham, 1986; Cowley et al., 1986; Cowley \& Atchley, 1988; M. Lynch \& J. B. Walsh,

Table 1 Expectations of the mean squares from the hierarchical analysis of variance in terms of their genetic causal components

\begin{tabular}{lccccc}
\hline & \multicolumn{5}{c}{ Causal components $\dagger$} \\
\cline { 2 - 6 } Mean square & $V_{\mathrm{A}}$ & $V_{\mathrm{D}}$ & $V_{\mathrm{AA}}$ & $V_{\mathrm{EC}}$ & $V_{\mathrm{EW}}$ \\
\hline Sires & $11 / 4$ & $3 / 2$ & $27 / 16$ & 3 & 1 \\
Dams & $5 / 4$ & $3 / 2$ & $21 / 16$ & 3 & 1 \\
Within progenies & $1 / 2$ & $3 / 4$ & $3 / 4$ & & 1 \\
Parent-offspring & $1 / 2$ & & $1 / 4$ & & \\
\hline
\end{tabular}

$\dagger$ The subscripts A, D, AA, EC and EW denote the respective components for additive, dominance, additiveby-additive epistasis, common environment and specific environment.

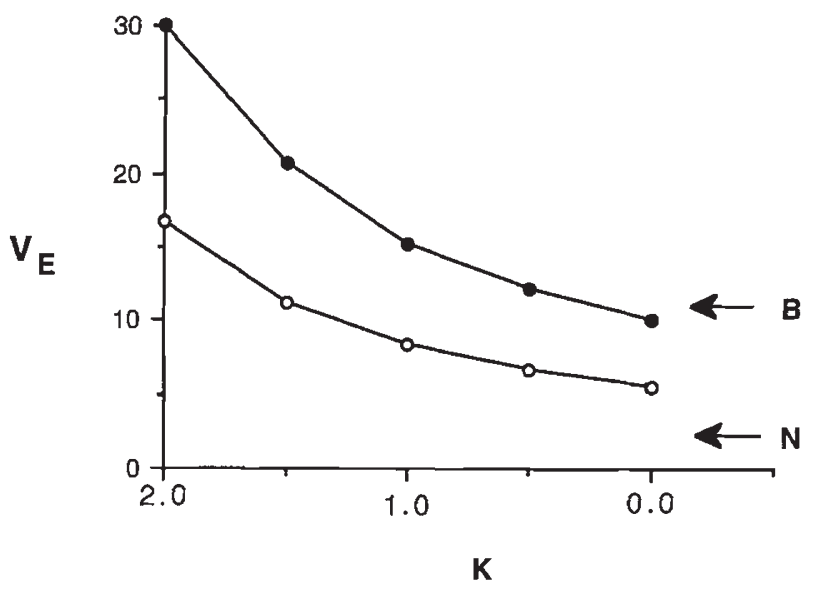

Fig. 1 Least squares estimates of the environmental variance for values of $k=V_{\mathrm{EW}} / V_{\mathrm{EC}}$ ranging from 2 (low common environment) to 0 (low specific environment), for the nonbottlenecked base population of houseflies $(O)$ and the average of the two bottleneck lines $(\bullet)$. Variance components observed among flies reared in different jars are indicated by arrows, where $\mathrm{N}$ and $\mathrm{B}$ are the nonbottlenecked and bottlenecked lines, respectively. unpublished ms.). The expected values of the mean squares in terms of the causal (genetic) components are shown in Table 1, which are derived directly from the expectations for the variance components (e.g. Becker, 1988), weighted by the contributions of the components to these mean squares. The vector (b) containing the estimates for the additive, dominance, epistatic and environmental components is given by

$\mathbf{b}=\left(\mathbf{X}^{t} \mathbf{V}^{-1} \mathbf{X}\right)^{-1} \mathbf{X}^{t} \mathbf{V}^{-1} \mathbf{Y}$

where ${ }^{t}$ and ${ }^{-1}$ indicate transpose and inverse, respectively, $\mathbf{X}$ is the matrix of design coefficients given in Table $1, \mathbf{Y}$ is a vector of mean squares for sires, dams, within progenies and parent-offspring, and $\mathbf{V}$ is a diagonal matrix whose elements are the variances of the mean squares (see Cowley \& Atchley, 1988, for a detailed discussion of this metholology).

With only four mean squares all potential causal components cannot be estimated. Maternal effects, for example, were omitted in these estimates. All females were reared on identical diets to eliminate diet as a potential source of these effects, and egg size in the housefly remains constant as female size varies, thus diminishing any differential yolk effects. Reciprocal crosses between selected and unselected lines from a similar base population did not show appreciable differences in means (Bryant \& Meffert, 1995), indicating no significant maternal effects. Nevertheless, the remaining five causal (genetic) components cannot be estimated with only four mean squares, so further consolidation of components was required. Therefore, the two environmental components arising from a common environment shared by full-sibs (i.e. the culture jar; $V_{\mathrm{EC}}$ ) and the specific (individual) environmental effect $\left(V_{\mathrm{EW}}\right)$ not shared by full-sibs (see Falconer, 1989) were collapsed into a single environmental component, $V_{\mathrm{E}}$, in the manner described below.

Setting $k=V_{\mathrm{EW}} / V_{\mathrm{EC}}$ we estimated the single environmental component, $V_{E}$, for $\mathrm{k}$ ranging from 0.0 to 2.0 , indicating a range from very small specific environment relative to the common environment to a large specific environmental component. The single environmental component, $V_{\mathrm{E}}$, estimated over the range of $k$ is shown in Fig. 1, showing the smallest $V_{\mathrm{E}}$ for small $k$ and the largest for large $k$. To determine an appropriate value for $k$ in our experiment an average variance component for the four traits among culture jars was estimated from 30 culture jars from each of the three populations. Assuming genotype-by-environment interactions to be negligible, the variance component among indivi- 
dual flies reared in different jars should approximate the culture jar effect across families in the experiment. These values are indicated (by arrows) in Fig. 1 , for the base population and the average of the two bottleneck lines, suggesting that estimates based on $k=0$ were the most appropriate, and this was used in subsequent variance component estimations.

Standard errors for the components of variance were obtained in two ways: from the square roots of the diagonal elements of $\left(\mathbf{X}^{t} \mathbf{V}^{-1} \mathbf{X}\right)^{-1}$ (Cowley \& Atchley, 1988; Lynch \& Walsh, unpublished ms.) and from 1000 bootstrap resamplings (with replacement) of families (Efron, 1982; Efron \& Gong, 1983). The bootstrap resamplings yielded estimates of the half-sib $\left(\sigma_{\mathrm{HS}}^{2}\right)$, full-sib $\left(\sigma_{\mathrm{FS}}^{2}\right)$ and parentoffspring $\left(\sigma_{\mathrm{PO}}^{2}\right)$ variance components in the analysis of variance. The variances for the genetic components $V_{\mathrm{A}}, V_{\mathrm{D}}$ and $V_{\mathrm{AA}}$ (additive, dominance, and additive-by-additive epistasis, respectively) were then obtained directly from linear functions of the respective variance components (Becker, 1988):

$V\left(V_{\mathrm{A}}\right)=64 V\left(\sigma_{\mathrm{HS}}^{2}\right)+4 V\left(\sigma_{\mathrm{PO}}^{2}\right)$,

$V\left(V_{\mathrm{D}}\right)=16 V\left(\sigma_{\mathrm{FS}}^{2}\right)+16 V\left(\sigma_{\mathrm{PO}}^{2}\right)$ and

$V\left(V_{\mathrm{AA}}\right)=64 V\left(\sigma_{\mathrm{PO}}^{2}\right)+256 V\left(\sigma_{\mathrm{HS}}^{2}\right)$, where the variances of the components, $V\left(\sigma_{\mathrm{HS}}^{2}\right)$, $V\left(\sigma_{\mathrm{FS}}^{2}\right)$ and $V\left(\sigma_{\mathrm{PO}}^{2}\right)$ were taken from the bootstrap resamplings. The (causal) components of phenotypic variance were deemed significant if either method yielded values significantly above zero (using a one-tailed probability).

\section{Results}

Estimates of the four variance components and the heritabilities based upon sire covariances are given in Table 2. In the nonbottlenecked (base) population, the heritabilities of the traits ranged from 0.20 to 0.42 , with an average over traits of 0.30 . Three of these heritabilities were significantly greater than zero (at $P<0.05$ ) and the remaining trait (metatibia) was marginally significant $(P<0.10)$. In contrast, heritabilities for the bottlenecked lines ranged from a low of 0.04 (metatibia; line $B_{1}$ ) to a high of 0.35 (wing length; line $\mathrm{B}_{2}$ ), and none of the heritabilities was significantly greater than zero. Nevertheless, the sample size for each of the bottlenecked lines was approximately one-half of that for the base population, reducing the power to detect significant heritabilities. Pooling over the two bottlenecked lines

Table 2 Estimates of causal components for the four traits and for the nonbottlenecked $(\mathrm{N})$ and two bottlenecked $\left(\mathrm{B}_{1}\right.$ and $\left.\mathrm{B}_{2}\right)$ populations of houseflies, along with narrow sense heritabilities based on the variance component among sires. All components are multiplied by $10^{4}$ for convenience

\begin{tabular}{llccccc}
\hline \multirow{5}{*}{ Trait } & & \multicolumn{5}{c}{ Causal component } \\
\cline { 3 - 6 } & Line & $V_{\mathrm{A}}$ & $V_{\mathrm{D}}$ & $V_{\mathrm{AA}}$ & $V_{\mathrm{E}}$ & Heritability \\
\hline \multirow{2}{*}{ Wing } & $\mathrm{N}$ & $7.16^{*}$ & 4.79 & 0.00 & $5.21^{*}$ & $0.42 \pm 0.13$ \\
& $\mathrm{~B}_{1}$ & 1.21 & 0.85 & 11.32 & $9.74^{*}$ & $0.20 \pm 0.24$ \\
& $\mathrm{~B}_{2}$ & $8.87^{*}$ & $9.95^{*}$ & 0.00 & $7.62^{*}$ & $0.35 \pm 0.18$ \\
& Mean (B) & $5.04^{*}$ & $5.40 \dagger$ & 5.66 & $8.68^{*}$ & $0.28 \pm 0.15$ \\
Inner-eye & $\mathrm{N}$ & $10.72^{*}$ & $15.86^{*}$ & $9.10 \dagger$ & $4.76^{*}$ & $0.33 \pm 0.11$ \\
& $\mathrm{~B}_{1}$ & $8.5 \mathbf{n}^{\dagger}$ & $17.70^{*}$ & 0.00 & $11.22^{*}$ & $0.20 \pm 0.19$ \\
& $\mathrm{~B}_{2}$ & 2.57 & 2.81 & $22.16^{*}$ & $10.75^{*}$ & $0.23 \pm 0.17$ \\
& Mean (B) & $5.58^{*}$ & $10.26^{*}$ & $11.08^{*}$ & $10.99^{*}$ & $0.21 \pm 0.13$ \\
Scutellum & $\mathrm{N}$ & 1.44 & 2.70 & $17.10^{*}$ & $6.81^{*}$ & $0.22 \pm 0.11$ \\
& $\mathrm{~B}_{1}$ & $8.94^{*}$ & 6.24 & 13.51 & $10.02^{*}$ & $0.33 \pm 0.22$ \\
& $\mathrm{~B}_{2}$ & 1.06 & 6.11 & 13.00 & $10.76^{*}$ & $0.15 \pm 0.18$ \\
& Mean (B) & $5.00^{*}$ & $6.17^{*}$ & $13.26^{*}$ & $10.39^{*}$ & $0.24 \pm 0.14$ \\
& $\mathrm{~N}$ & 1.43 & 2.15 & $8.84 \dagger$ & $3.86^{*}$ & $0.20 \pm 0.11$ \\
& $\mathrm{~B}_{1}$ & 0.00 & 3.64 & 11.13 & $10.02^{*}$ & $0.04 \pm 0.23$ \\
& $\mathrm{~B}_{2}$ & $5.47^{*}$ & $10.27^{*}$ & 0.00 & $8.33^{*}$ & $0.22 \pm 0.19$ \\
& Mean (B) & 2.73 & $6.96^{*}$ & 5.57 & $9.18^{*}$ & $0.13 \pm 0.15$ \\
Means & $\mathrm{N}$ & $5.19^{*}$ & $6.38^{*}$ & $8.76^{*}$ & $5.16^{*}$ & $0.30 \pm 0.11$ \\
& $\mathrm{~B}$ & $4.59^{*}$ & $7.20^{*}$ & $8.89^{*}$ & $9.81^{*}$ & $0.22 \pm 0.14$ \\
\hline
\end{tabular}

$\dagger P<0.10 ;{ }^{*} P<0.05$. 
yielded an average sample size comparable to that of the base population (364 sires versus 431 sires, respectively), resulting in a range of heritabilities over the four traits from 0.13 to 0.28 ; only one of these heritabilities was marginally significant from zero (at $P<0.10$ ). Over all traits, the average narrow sense heritability was not significantly different between bottlenecked and nonbottlenecked populations.

Each component of phenotypic variance for the three populations was significant for at least one of the traits, so that all components of variation were significantly different from zero when averaged over the four traits $(P<0.05$, Table 2 , bottom). The average of the additive components of variance for the bottlenecked lines $(=4.59)$ was 88 per cent of that for the base population $(=5.19)$, which was very close to the expected value of 87.5 per cent obtained from $1-1 / 2 N$, where $N$ is the bottleneck size of four individuals. The average environmental contribution to phenotypic variance in the bottlenecked lines $(=9.81)$ was significantly greater than that in the nonbottlenecked base population $(=5.16)$, reflecting apparently greater phenotypic plasticity for these traits after the bottleneck. The remaining averages for the dominance and epistatic components were remarkably similar between the nonbottlenecked and bottlenecked treatments.

Despite the similarity of average genetic components in the nonbottlenecked base and bottlenecked lines, there was considerable variation among traits in the contributions of individual components to phenotypic variance, as well as variation among traits in total phenotypic variance. To facilitate direct comparisons among traits, components in Table 2 were scaled as percentages of phenotypic variances for each trait in Fig. 2. The contribution of the additive component to phenotypic variation in the nonbottlenecked population ranged from 5.1 per cent (scutellum length) to 41.7 per cent (wing length). The percentage contribution of nonadditive genetic variance (dominance and epistasis together) ranged from 28 per cent of phenotypic variance (wing length) to 71 per cent (scutellum length). For the bottlenecked lines, additive genetic variance ranged from 11 per cent (metatibia) to 20 per cent (wing length), whereas the percentage of nonadditive genetic variance was very close among traits, ranging from 45 per cent (wing) to 56 per cent (inner-eye).

In comparison with the nonbottlenecked population, additive genetic variance for the bottlenecked populations declined for wing and inner-eye but increased for scutellum and metatibia. Both the decrease for inner-eye and the increase for scutellum were significantly different from zero (at $P<0.05$ ), indicating that a bottleneck can create bidirectional changes in additive genetic variances among a suite of traits (see also Meffert, 1995). The traits exhibiting increases in the additive component of variance, however, also exhibited larger epistatic components in the base population, and vice versa. Goodnight's (1988) model predicts such a relationship between the magnitude of the initial epistatic
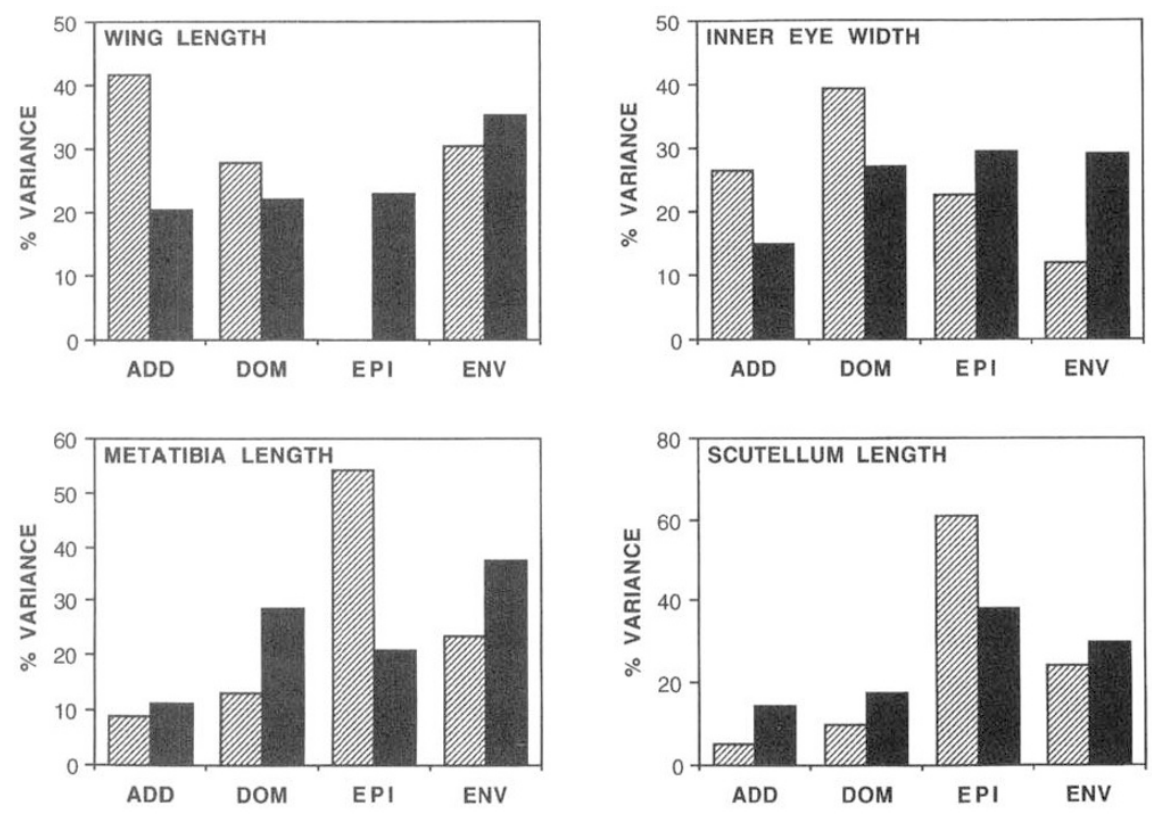

Fig. 2 Percentage contribution of the components for additivity (ADD), dominance (DOM), additive-by-additive epistasis (EPI) and environment (ENV) to the phenotypic variance for each trait, for the nonbottlenecked base population of houseflies (hatched bars) and bottlenecked lines (solid bars).

(c) The Genetical Society of Great Britain, Heredity, 77, 168-176. 
component in a base population and the gain in the additive component after a bottleneck.

To investigate Goodnight's prediction in our data, Fig. 3 plots the ratio of the additive genetic variance in the bottlenecked lines to that of the nonbottlenecked base population against the ratio of the epistatic to the additive genetic variance components in the nonbottlenecked base population, for the experimental results and for predictions from Goodnight's model (see Goodnight, 1988, 1995). When the influence of additive-by-additive epistasis on a trait was greater in the base population, there was a greater increment in the additive component after a bottleneck, as predicted by the model. The response was significant by linear regression at $P<0.01$ (as the traits represented essentially independent axes of morphometric variation, the average squared additive genetic correlation between traits was only 0.13 , justifying the use of the four traits as independent estimates in regression). However, the resultant slope of regression $(b=0.25)$ was only half of the expected slope $(b=0.45)$. Futhermore, the data failed to fit the predicted response mostly for the two traits with large epistatic components, so that the observed gain in additive genetic variance for these traits was considerably lower than predicted by the model. As a result, Goodnight's model predicts that additive genetic variance in the bottlenecked lines should exceed that in the base population as the ratio of epistatic to additive genetic variance rises above 0.33 , whereas for our results the critical ratio was approximately 2.0 , indicating that substan-

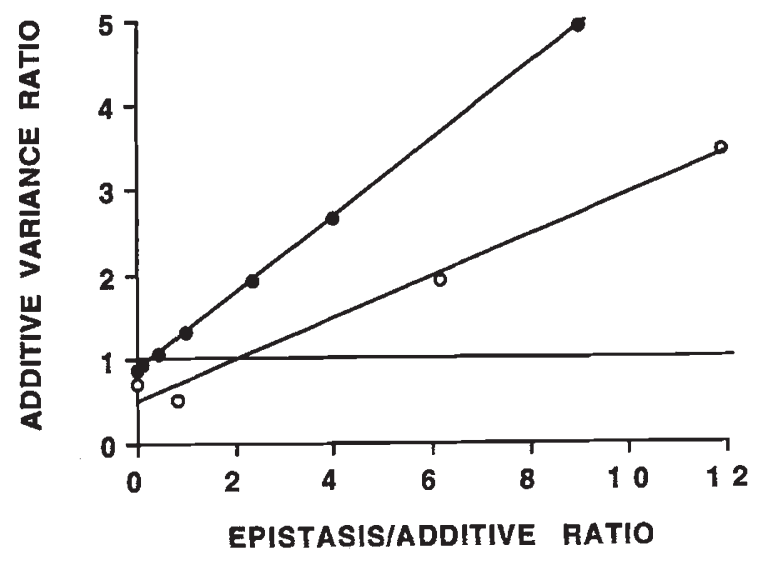

Fig. 3 The ratio of the additive genetic variance in the bottleneck lines of houseflies relative to that in the ancestor (ordinate) in relation to the ratio of epistasis to additive components of variance in the nonbottlenecked base population (abscissa). Empirical estimates $(0)$ and expectations from Goodnight (1988) (•) are shown. tially higher levels of epistasis may be required before effecting an increase in the additive genetic variance after a bottleneck.

\section{Discussion}

Additive genetic variance after a bottleneck increased for two of the traits and decreased for the remaining two, resulting in an overall decline in average additive genetic variance and narrow sense heritability after the bottleneck. This contrasts with our previous results reporting an increase in the average additive genetic variance for morphometric traits after a bottleneck (Bryant et al., 1986; Bryant \& Meffert, 1993). Nevertheless, wing length in Bryant et al. (1986) did not show an increase in the additive component of variance for the smallest bottleneck size of one pair, which was consistent with the results of the two-pair bottleneck here. Furthermore, scutellum length exhibited the largest increase in the additive component in both experiments. Therefore, differences in the average performance of the additive genetic variance depended on the particular suite of traits assayed (eight traits were assayed in Bryant et al., 1986, compared to only four traits here), and not necessarily upon any major disparity in results.

We found a surprisingly large influence of nonadditive genetic variance on morphometric variation in the housefly, and it is important to know how general these results might be. Unfortunately, there is a paucity of evidence for epistasis affecting quantitative traits in other organsisms (e.g. Jones et al., 1968; Barker, 1979; Webber, 1990a,b; Coyne \& Beecham, 1987; but see Miller et al., 1963; Kearsey \& Kojima, 1967). These negative results, however, may reflect the low statistical power for detecting epistasis in most designs (e.g. Shaw, 1987). Our analysis of more than 2400 flies on average per population may have increased the power to detect nonadditive components, and this study is unusual in incorporating parent-offspring covariances into a standard half-sib design to allow for estimates of epistasis as well as dominance.

With the advent of QTL methods, the opportunity for detecting digenic interactions may be greater, because interactions between marker loci can be examined directly (Crow \& Kimura, 1970). Cheverud \& Routman (1995) utilized this approach to show large epistatic effects between loci affecting body weight in mice, and, similarly, Lark et al. (1995) demonstrated strong interactions among loci affecting plant height in soybean. Long et al. (1995) found interactions among QTL factors for abdomi- 
nal bristle number in Drosophila melanogaster to be equally important as additive effects in determining bristle number variation. This latter result is particularly striking because abdominal bristle number in Drosophila has been historically regarded as highly additive (e.g. Falconer, 1989). These results suggest that epistasis may be more prevalent than previously considered and the paucity of direct evidence for epistasis on quantitative traits may originate more from lack of statistical power than from the absence of epistasis (but see Shrimpton \& Robertson, 1988; Patterson et al., 1991). Nevertheless, the observation that many quantitative traits are normally distributed argues against large influences of nonadditive effects (Barton \& Turelli, 1989; Turelli \& Barton, 1990). However, the resulting distribution of individual traits may be nearly normal despite underlying epistasis if loci interact to form groups of functionally inter-related loci (Wright, 1932; Moreno, 1994) whose combined effects are themselves additive.

One unusual aspect of our experimental protocol was the use of newly established populations, for which evolutionary adjustments to the laboratory environment may have been concurrent with our experiments. Moreno (1994) recently argued that many quantitative traits in equilibrium populations (such as those well established in the laboratory) would exhibit little epistasis, because of a nonlinear relationship of genotype to phenotype caused by redundancy in phenotypic effects among loci within developmentally co-ordinated suites of loci (see also Keightly, 1989). When populations are genetically perturbed, through, for example, inbreeding, selection and/or bottlenecks, dramatic changes in some alleles within a functional set may cause previously cryptic interaction effects to emerge. Such a factor could have enabled our results, although we do not have direct evidence for it.

An important result of this study was the relationship between the magnitude of epitasis affecting a trait in the base population and the increase in additive component for the trait after a bottleneck (predicted by Goodnight, 1987, 1988). Although this seems to implicate epistasis as the root cause of increased additive genetic variance after a bottleneck, dominance can also cause similar effects (Robertson, 1952; Willis \& Orr, 1993). Indeed, there was substantial dominance contribution to trait variation. However, there was no clear relationship between the initial level of dominance for a trait and the gain in the additive component after the bottleneck as there was for epistasis (e.g. see wing length), arguing against a primary role of directional dominance as causing the increased additive genetic vari- ance in bottlenecked lines. Nevertheless, the increase in the additive component of variance in our experiment was clearly less than predicted by Goodnight's model for additive-by-additive epistasis (Fig. 3). However, our genetic components were estimated sometime after the initial bottleneck, when populations had grown to large size. Any selection occurring during the flush phase before assays for genetic variance were carried out could have reduced additive genetic variance. Thus, the actual gain in the additive component in our experiments could well have been as large as predicted but it was ameliorated by selection before testing occurred.

The most striking result of the study was the considerable nonadditivity affecting morphometric variation, amounting to 75 per cent of the genetic variance in the base population, with roughly half of this attritutable to epistasis. In a separate study on the genetic basis of selectional response in morphometric shape, we also found considerable nonadditive contributions, amounting to 80 per cent of the phenotypic response (Bryant \& Meffert, 1995), with over half of the response effected by epistasis. These results offer support to our previous conclusion that epistasis was responsible for the observed increases in the additive genetic variance for these traits after a bottleneck (Bryant, et al., 1986; Bryant \& Meffert, 1993). Taken together the studies offer strong evidence that nonadditive components affect morphometric variation in outbred populations of the housefly and that these components can directly influence the way populations respond to selection, particularly in small populations.

\section{Acknowledgments}

Support for this study was supplied through grants from The National Science Foundation to E. H. Bryant (BSR 8800977 and BSR 9024980) and L. M. Meffert (BSR 9106591) and through the University of Houston Coastal Center. We thank Charles J. Goodnight, William R. Atchley and two anonymous reviewers for their valuable comments on an earlier draft of the manuscript.

\section{References}

BARKER, J. S. F. 1979. Inter-locus interactions: a review of experimental evidence. Theor. Pop. Biol., 16, 323-346.

BARTON, N. H. AND TURELLI, M. 1989. Evolutionary quantitative genetics: how little do we know? Ann. Rev. Genet., 23, 337-370.

BECKER, w. A. 1988. Manual of Quantitative Genetics. 
Academic Enterprises, Pullman, WA.

BRYANT, E. H. 1977. Morphometric adaptation of the housefly, Musca domestica L., in the United States. Evolution, 31, 580-596.

BRYANT, E. H. 1986. On the use of logarithms to accommodate scale. Syst. Zool., 35, 552-559.

BRYANT, E. H: AND MEFFERT, L. M. 1988. Effect of an experimental bottleneck on morphological integration in the housefly. Evolution, 42, 698-707.

BRYANT, E. H. AND MEFFERT, L. M. 1990. Multivariate phenotypic differentiation among bottleneck lines of the housefly. Evolution, 44, 660-668.

BRYANT, E. H. AND MEFFERT, L. M. 1993. The effect of serial founder-flush cycles on quantitative genetic variation in the housefly. Heredity, 70, 122-129.

BRYANT, E. H. AND MEFFERT, L. M. 1995. Analysis of selectional response in relation to a population bottleneck. Evolution, 49, 626-634.

BRYANT, E. H., McCOMMAS, S. A. AND COMBS, L. M. 1986. The effect of an experimental bottleneck upon quantitative genetic variation in the housefly. Genetics, 114, 1191-1211.

CHARLESWORTH, D. AND CHARLESWORTH, B. 1987. Inbreeding depression and its evolutionary consequences. Ann. Rev. Ecol. and Syst., 18, 237-268.

CHEVERUD, J. M. AND ROUTMAN, E. J. 1995. Epistasis and its contribution to genetic variance components. Genetics, 139, 1455-1461.

COCKERHAM, C. C. 1956. Estimation of genetic variances. Statistical genetics and plant breeding. National Academy of Science, U.S.A., Publication Number 982.

COCKERHAM, C. C. 1986. Modifications in estimating the number of genes for a quantitative character. Genetics, 114, 659-664.

COCKERHAM, C. C. AND TACHIDA, H. 1988. Permanancy of response to selection for quantitative characters in finite populations. Proc. Natl. Acad. Sci. U.S.A., 85, 1563-1565.

COWLEY, D. E. AND ATCHLEY, W. R. 1988. Quantitative genetics of Drosophila melanogaster. II. Heritabilities and genetic correlations between sexes for head and thorax traits. Genetics, 119, 421-433.

COWLEY, D. E., ATCHLEY, W. R. AND RUTLEDGE, J. J. 1986. Quantitative genetics of Drosophila melanogaster. I. Sexual dimorphism in genetic parameters for wing traits. Genetics, 114, 549-566.

COYNE, J. A. AND BEECHAM, E. 1987. Heritability of two morphological characters within and among natural populations of Drosophila melanogaster. Genetics, 117, 727-737.

Crow, J. F. AND kImURa, м. 1970. An Introduction to Population Genetics Theory. Harper and Row, New York.

DRAPER, N. R. AND SMITH, N. M. 1981. Applied Regression Analysis. John Wiley, New York.

EFRON, B. 1982. The Jackknife, the Bootstrap and Other Resampling Plans. Society for Industrial and Applied Mathematics, Philadelphia, PA.

EFRON, B. AND GONG, G. 1983. A leisurely look the bootstrap, the jackknife, and cross-validation. Am. Statist.,
37, 36-48.

FALCONER, D. s. 1989. Introduction to Quantitative Genetics, 3rd edn. John Wiley, New York.

GIMELFARB, A. 1989. Genotypic variation for a quantitative character maintained under stabilizing selection without mutations: epistasis. Genetics, 123, 217-227.

GOODNIGHT, c. J. 1987. On the effect of founder events on epistatic genetic variance. Evolution, 41, 171-179.

GOODNIGHT, C. J. 1988. Epistasis and the effect of founder events on the additive genetic variance. Evolution, 42, 441-454.

GOODNIGHT, C. J. 1995. Epistasis and the increase in additive genetic variance: implications for Phase I of Wright's shifting balance process. Evolution, 49, 502-511.

HARD, J. J., BRADSHAW, W. E. AND HOLZAPFEL, C. M. 1992. Epistasis and the genetic divergence of photoperiodism between populations of the pitcher-plamt mosquito, Wyeomyia smithii. Genetics, 131, 389-396.

HASTINGS, A. 1989. Linkage disequilibrium and genetic variances under mutational-selection balance. Genetics, 121, 857-860.

HEDRICK, P. H., JAIN, S. AND HOLDEN, L. 1978. Multilocus systems in evolution. Evol. Biol., 11, 101-182.

JONES, L. S., FRANKHAM, R. AND BARKER, J. S. F. 1968. The effects of population size and selection intensity in selection for a quantitative character in Drosophila. II. Long-term response to selection. Genet. Res., 12, 249-264.

KEARSEY, M. J. AND KOJIMA, к. 1967. The genetic architecture of body weight and egg hatchability in Drosophila melanogaster. Genetics, 56, 23-37.

KEIGHTLY, P. D. 1989. Models of quantitative variation in flux in metabolic pathways. Genetics, 121, 869-876.

LANDER, E. AND BOTSTEIN, D. 1989. Mapping Mendelian factors underlying quantitative traits using RFLP linkage maps. Genetics, 121, 185-199.

LARK, K. G., CHAES, K., ADLER, F., MANSUR, L. M. AND ORF, J. H. 1995. Interactions between quantitative trait loci in soybean in which trait variation at one locus is conditional upon a specific allele at another. Proc. Natl. Acad. Sci. U.S.A., 92, 4656-4660.

LONG, A. D., MULLANEY, S. L, REID, L. A., FRY, J. D., LANGLEY, C. H. AND MACKAY, T. F. C. 1995. High resolution mapping of genetic factors affecting abdominal bristle number in Drosophila melanogaster. Genetics, 139, 1273-1291.

LUSH, J. L. 1940. Intra-sire correlations or regressions of offspring on dam as a method of estimating heritability of characters. Proceedings of the American Society of Animal Production, 33, 293-301.

MATHER, K AND JINKS, J. L. 1982. Biometrical Genetics, 3rd edn. Chapman and Hall, New York.

MEFFERT, L. M. 1995. Bottleneck effects on genetic variance for courtship repertoire. Genetics, 139, 365-374.

MILLER, R. H., LEGATES, J. E. AND COCKERHAM, C. C. 1963. Estimation of nonadditive hereditary variance in traits of mice. Genetics, 48, 177-188.

MORENO, G. 1994. Genetic architecture, genetic behavior,

(C) The Genetical Society of Great Britain, Heredity, 77, 168-176. 
and character evolution. Ann. Rev. Ecol. Syst., 25, 31-44.

NIE, N. H., HULL, C. H., JENKINS, J. G., STEINBRENNER, K. AND BENT, D. H. 1975. SPSS: Statistical Package for the Social Sciences. McGraw-Hill, New York.

PATERSON, A. H., DAMON, S., HEWITT, J. D., ZAMIR, D., RABINOWITCH, H. D., LINCOLN, S. E., LANDER, E. S. AND TANKSLEY, S. D. 1991. Mendelian factors underlying quantitative traits in tomato: comparison across species, generations, and environments. Genetics, 127, 181-197.

RISKA, B., RUTLEDGE, J. AND ATCHLEY, w. R. 1985. Genetic analysis of crossfostering data with sire and dam records. $J$. Hered., 76, 247-250.

ROBERTSON, A. 1952. The effect of inbreeding on the variation due to recessive genes. Genetics, 37, 189-207.

robertson, A. 1955. Selection in animals: synthesis. Cold Spring Harb. Symp. Quant. Biol., 20, 225-229.

ROBERTSON, A. 1967. The nature of quantitative genetic variation. In: Brink, R. A. (ed.) Heritage from Mendel, pp. 265-280. University of Wisconsin Press, Madison, WI.

ROBERTSON, F. W. 1954. Studies in quantitative inheritance. V. Chromosome analysis of crosses between selected and unselected lines of different body size in Drosophila. J. Genet., 52, 494-520.

ROBERTSON, F. W. 1955. Selectional response and the properties of genetic variation. Cold Spring Harb. Symp. Quant. Biol., 20, 166-177.

SHAW, R. G. 1987. Maximum-likelihood approaches to quantitative genetics of natural populations. Evolution, 41, 812-826.
SHRIMPTON, A. E. AND ROBERTSON, A. 1988. The isolation of polygenic factors controlling bristle score in Drosophila melanogaster. I. Allocation of third chromosome bristle effects within chromosome sections. Genetics, 118, 437-443.

TACHIDA, H. AND COCKERHAM, C. C. 1989a. Effects of identity disequilibrium and linkage on quantitative variation in finite populations. Genet. Res., 53, 63-70.

TACHIDA, H. AND COCKERHAM, C. C. 1989 b. A building block model for quantitative genetics. Genetics, 121, 247-262.

TURElli, M. AND BARTON, N. 1990. Dynamics of polygenic characters under selection. Theor. Pop. Biol., 38, 1-57.

WEBBER, K. E. 1990a. Increased selectional response in larger populations. I. Selection for wing-tip height in Drosophila melanogaster at three population sizes. Genetics, 125, 579-584.

WEBBER, K. E. 1990b. Selection on wing allometry in Drosophila melanogaster. Genetics, 126, 975-989.

WILLIS, J. H. AND ORR, H. A. 1993. Increased heritability variation following population bottlenecks: the role of dominance. Evolution, 47, 949-957.

WRIGHT, s. 1931. Evolution in Mendelian populations. Genetics, 16, 97-159.

WRIGHT, s. 1932. General, group, and special size factors. Genetics, 17, 603-619.

WRIGHT, s. 1935. Evolution of populations in approximate equilibrium. J. Genet., 30, 257-266.

WRIGHT, s. 1940. Breeding structure of populations in relation to speciation. Am. Nat., 74, 232-248. 